\title{
Téoros
}

Revue de recherche en tourisme

\section{Découvertes pour tous}

Les paysages se racontent

\section{Jocelyn Caron}

Volume 19, numéro 1, printemps 2000

Pour une culture du tourisme au Saguenay-Lac-Saint-Jean

URI : https://id.erudit.org/iderudit/1071797ar

DOI : https://doi.org/10.7202/1071797ar

Aller au sommaire du numéro

Éditeur(s)

Université du Québec à Montréal

ISSN

0712-8657 (imprimé)

1923-2705 (numérique)

Découvrir la revue

Citer cet article

Caron, J. (2000). Découvertes pour tous : les paysages se racontent. Téoros,

19(1), 5-8. https://doi.org/10.7202/1071797ar

Ce document est protégé par la loi sur le droit d'auteur. L'utilisation des services d'Érudit (y compris la reproduction) est assujettie à sa politique d'utilisation que vous pouvez consulter en ligne.

https://apropos.erudit.org/fr/usagers/politique-dutilisation/
Cet article est diffusé et préservé par Érudit.

Érudit est un consortium interuniversitaire sans but lucratif composé de l'Université de Montréal, l'Université Laval et l'Université du Québec à Montréal. Il a pour mission la promotion et la valorisation de la recherche. https://www.erudit.org/fr/ 


\section{Découvertes pour tous}

LES PAYSAGES SE RACONTENT

\section{Jocelyn Caron}

L'observation des paysages doit dépasser la seule appréciation visuelle. L'ajout d'information pertinente permet à l'observateur d'acquérir des connaissances variées et de mieux apprécier le milieu où il se trouve. L'une des tendances actuelles de la demande touristique indique que les individus désirent apprendre tout en voyageant. Les paysages sont dépositaires autant de l'histoire naturelle que de l'histoire humaine du territoire. C'est dans cet esprit que s'inscrit la présente description de certains paysages de la région du Saguenay-Lac-Saint-Jean. Ils ont été sélectionnés en fonction de leur accessibilité, de leur diversité et de l'intérêt qu'ils suscitent chez les observateurs.

Au plan géographique, la région du Saguenay-Lac-Saint-Jean présente trois grandes unités écologiques qui forment et caractérisent les paysages régionaux. Ce sont la plaine du lac Saint-Jean, le Haut-Saguenay et le fjord du Saguenay. Les nombreux paysages naturels qu' on y trouve offrent un spectacle souvent exceptionnel. Quant aux paysages ruraux et urbains, bien que moins spectaculaires, ils reflètent et témoignent de l'action de l'homme. Ces paysages « humanisés » évoluent et changent continuellement.

Le voyageur qui visite le Saguenay-LacSaint-Jean pour la première fois est frappé par la présence de cours d'eau de grande dimension : le lac Saint-Jean, la rivière Saguenay et le lac Kénogami constituent d'ailleurs des incontournables du circuit touristique régional. Il remarque également une grande variété de contrastes entre les paysages tout en douceur de la cuvette du lac Saint-Jean et les formes escarpées du fjord du Saguenay. Il peut observer et profiter de ces paysages de multiples façons : que ce soit lors de déplacements en automobile, à l'occasion de randonnées pédestres, de balades à vélo ou encore de croisières en bateau, les nombreux points de vue rencontrés sont autant d'incitatifs à la découverte et à l'exploration de la région.

\section{LES PAYSAGES APPRIVOISÉS DU LAC SAINT-JEAN}

La présence de la cuvette du lac Saint-Jean au cœur du massif laurentien peut parầtre bien étrange aux visiteurs. L' histoire géologique de la région nous apprend que cette enclave aurait été causée par une rupture importante de la croûte terrestre. Il y a 180 millions d'années, un fossé d'effondrement s'est formé ; délimité par deux énormes failles taillées dans le granit, il a donné naissance à une dépression occupée par les basses terres du Lac-SaintJean et du Saguenay.

C'est au cours du dernier million d'années que l'action érosive des glaciers a permis de compléter le façonnage du paysage et de lui donner sa forme actuelle. Les mar- ques des glaciations successives sont nombreuses et faciles à observer. En traversant la plaine agricole de Saint-Bruno, en direction de Jonquière, on remarque aisément des roches moutonnées. Ces affleurements rocheux polis et arrondis témoignent de la dernière glaciation. Il en est de même des kettles, ces «trous » laissés par les glaciers peuvent atteindre plus de 100 mètres de diamètre. Dans le secteur de Métabetchouan-Lac-à-la-Croix et Hébertville, on en dénombre environ 300 , une concentration unique au Québec. En plein cœur de Lac-à-la-Croix, on peut examiner tout à loisir un bel exemple de kettle à fond sec.

Au sud du lac Saint-Jean, les montagnes arrondies du contrefort des Laurentides marquent la limite de l'une des deux failles. Elles entourent une étroite plaine qui ceinture les eaux du lac Saint-Jean, offrant un paysage plutôt rural et agricole. Les terres fertiles sont des vestiges laissés par la mer de Laflamme qui, à son maximum, couvrait sept fois la surface actuelle du lac Saint-Jean. Cette mer a déposé, lors de son retrait, plus de 20 mètres d'argile, de limon et de sable fin, formant les plaines dites argileuses. Pour observer facilement cette magnifique plaine quadrillée de champs, il s'agit, à partir de Québec, d'emprunter la route 169 en direction d'Alma. Dès qu'on arrive dans la région, on a une vue imprenable sur la plaine d'Hébertville et sur un bel exemple de paysage modelé par des activités agricoles. Depuis quelques années d'ailleurs, le Centre de ski du mont Lac-Vert, du haut de ses 300 mètres, propose des activités qui visent à tirer profit de la vue magnifique que l'on a du sommet de la montagne. 
C'est en 1647 que le jésuite Jean de Quen nous a fourni la première véritable description du lac Saint-Jean; il a vanté les ressources et les splendides paysages qu'il a remarqués. Ce lac, l'un des plus grands du Québec, impressionne toujours autant par son immensité. Son caractère grandiose lui donne l'aspect d'une véritable mer intérieure. Il est aussi large que le fleuve SaintLaurent à la hauteur de Tadoussac. D'une superficie de $1000 \mathrm{~km}^{2}$ et pourvu de $210 \mathrm{~km}$ de rives, il présente une multitude de paysages. Les points de vue sont nombreux et saisissants. Un arrêt à l'une ou l'autre des deux haltes routières situées sur la route 169 , l'une à Métabetchouan et l'autre près de Chambord, donne un avantgoût du spectacle. Les vues superbes, à partir du Camp musical de Métabetchouan ou du haut de la passerelle de la chute de Val-Jalbert, dévoilent le lac Saint-Jean dans toute sa splendeur. Tout en visitant les installations du village historique de ValJalbert, on remarque que la chute coule le long d'un mur rocheux très résistant à l'érosion. Cet escarpement est d'ailleurs l'un des indices confirmant la présence de la grande faille qui longe la partie sud du lac Saint-Jean.

Depuis peu, la Véloroute des Bleuets, circuit cyclable de plus de $250 \mathrm{~km}$ qui ceinture le lac Saint-Jean, fournit l'occasion de redécouvrir les paysages de ses rives et d'en apprécier de nouveaux. Ce circuit traverse 19 municipalités et propose un lien interactif dynamique entre les attraits naturels et humains qu' on y trouve. Le tronçon situé entre Métabetchouan et Desbiens révèle des panoramas éblouissants mettant en vedette l'immensité du lac et les falaises d' argile de Desbiens. De même, entre Val-Jalbert et Roberval, le lac et ses paysages se déploient sur plusieurs kilomètres. Les utilisateurs de la Véloroute ont accès à de nombreux sites d'intérêt, dont le parc de la Pointe-Taillon qui possède un circuit cyclable donnant accès directement à la plus grande plage du lac Saint-Jean. L'abondance de sable distingue d'ailleurs le lac Saint-Jean des autres plans d'eau québécois. Près d'un cinquième de ses berges, soit 42 kilomètres, sont des plages de sable s'étendant souvent sur plusieurs kilomètres, dévoilant des vues où de longues bandes de sable s'entremêlent à l'eau. La coloration du sable varie selon les endroits. Par exemple, dans le secteur du parc de la Pointe-Taillon, il contient des grains d'ilménite (oxyde de fer et de titane) qui lui donnent une teinte foncée ; à Saint-Gédéon, sa coloration dorée est due à la présence de grains de quartz et à Chambord, c'est un mélange de grenat et d'ilménite qui lui donne sa pigmentation rougeâtre.

La navigation sous toutes ses formes permet de se remémorer l'histoire des villages et des villes qui bordent ses rives. De 1870 à 1910 , une soixantaine de bateaux d'une petite marine marchande circulaient sur les eaux du lac Saint-Jean pour approvisionner les villages qui s'y installaient. Chaque village avait son quai où l'on débarquait pêle-mêle passagers, animaux, bois et marchandises de toutes sortes. Les croisières en bateau sont un excellent moyen de bien saisir toute l'ampleur du lac Saint-Jean. Le nouveau lien maritime entre Alma et Roberval, à bord du bateau La Tournée, donne l'occasion de découvrir le lac et de s'en imprégner pleinement.

Quand on s'éloigne du lac Saint-Jean et de sa plaine agricole pour pénétrer à l'intérieur de la forêt boréale, ce sont les paysages naturels qui s'imposent. Les trois grandes rivières forestières du nord que sont la Péribonka, la Mistassini et 1'Ashuapmushuan fournissent $90 \%$ des apports d'eau du lac. Elles charrient, à elles seules, l'équivalent de 3000 wagons de sable par année. La rivière Ashuapmushuan, l'une des grandes rivières sauvages du Québec, invite à l'aventure comme à l'époque des premiers explorateurs. Ses nombreuses chutes (à Michel, à l'Ours, Chaudière) impressionnent et suscitent l'admiration. À partir de SaintFélicien, on a la chance de côtoyer cette rivière extraordinaire considérée comme le paradis des amateurs de plein air.

\section{LES PAYSAGES URBANISÉS DU HAUT-SAGUENAY}

Le territoire du Haut-Saguenay se situe entre le lac Saint-Jean et la baie des $\mathrm{Ha}$ ! Ha ! Il comprend l'agglomération urbaine la plus importante et la plus concentrée de la région (Chicoutimi-Jonquière). Bien que le territoire soit fortement urbanisé, on en fait avant tout une utilisation forestière ; l'agriculture occupe aussi une bonne part. Le relief est relativement doux et percé par de nombreuses buttes et quelques ensembles de collines. En circulant entre SaintBruno et Jonquière, on franchit aisément l'un de ces ensembles au relief moutonné qui forme une barrière naturelle entre le lac Saint-Jean et le Saguenay qu'on appelle « horst de Kénogami ».

Sur le plan physique, la rivière Saguenay domine le paysage. De son lieu de naissance, la Pointe-des-Américains à Alma, jusqu'à La Baie, on peut s'imprégner des beautés de cette grande rivière. À l'exception du charmant petit village de SaintCharles-de-Bourget qui en offre une vision campagnarde, ce sont les paysages urbains et industriels qui bordent les rives les plus accessibles. Ces paysages reflètent les modifications apportées par 1'action de l'homme. Avec le temps, certaines des transformations s'intègrent mieux à l'environnement visuel. C'est le cas des grands ouvrages hydroélectriques qui sont à la base du développement économique de la région et qui constituent des paysages industriels remarquables. Une visite sur les sites entourant les centrales d'IsleMaligne et de Shipsaw permet d'apprécier l'ampleur des travaux réalisés et les changements apportés à la rivière. À l'époque, il s'agissait de travaux gigantesques, comparables au récent développement des rivières Manicouagan et La Grande.

La production d'électricité en abondance a d'ailleurs permis l'implantation de grandes entreprises des secteurs de l'aluminium et du papier. Ces usines sont à la base de la configuration et de l'organisation de plusieurs villes du Saguenay. À certains endroits, l'établissement de grandes entreprises améliore la qualité du paysage, tandis qu'à d'autres, les perturbations sont majeures. À La Baie, par exemple, l'usine de pâte et papier s'est installée en plein centre de la Baie des $\mathrm{Ha}$ ! Ha ! bloquant ainsi, en bonne partie, la vue imposante sur la rivière Saguenay. À Jonquière, il en a été tout autrement. L'usine d'aluminium est construite sur un plateau et on y a aménagé à proximité un modèle exemplaire et avant-gardiste de ville de compagnie. Baptisé du nom du président de l'Aluminium Company of America de l'époque, ARthur VIning DAvis, et édifiée en 135 jours, Arvida devait fournir aux travailleurs des logements modernes tout en leur offrant un milieu de vie de qualité. Maintenant intégrée à la ville de Jonquière, Arvida a su conserver son caractère d'origine, préserver son urbanisme si particulier ainsi que l'harmonie de son architecture. À titre de curiosité, en 1939 on y a construit le Manoir du Saguenay, magnifique édifice à l' architecture traditionaliste qui 
s'inspire, vraisemblablement, du manoir de Jacques-Cartier bâti près de la ville de Saint-Malo. La reconnaissance de l'ensemble du secteur d'Arvida comme patrimoine urbain unique en Amérique du Nord fera d'ailleurs l'objet d'une demande auprès de l'Unesco.

L'influence de la grande industrie est peu marquée à Chicoutimi. Ce sont plutôt les grandes institutions (religion, éducation, santé) qui lui ont donné naissance. Installée de part et d'autre de la rivière Saguenay, la ville est construite par paliers successifs, suivant les mouvements ondulés du terrain. En empruntant les rues vallonnées de ses anciens quartiers, certains vont même jusqu'à la qualifier de «SanFrancisco du Nord ». À partir de la rive nord, la vue en plongée sur le Centre procure un panorama harmonieux où toutes les pièces d'un puzzle architectural s'imbriquent parfaitement les unes aux autres. Une impression d'homogénéité se dégage de cet ensemble urbain. Les différentes époques se superposent comme les cou- ches de sable servant à définir l'histoire géologique d'un lieu.

Les inondations de 1996, qu'on désigne communément par « le déluge », fournissent une image puissante de l'impact des éléments naturels sur un paysage urbain. À Chicoutimi, la force de l'eau et son action érosive extrêmement puissante ont entraîné des milliers de tonnes de sédiments et provoqué le décapage en profondeur du sol, bouleversant de fond en comble le vieux quartier du Bassin. Ce quartier ouvrier s'était développé, au début du $\mathrm{XX}^{\mathrm{e}}$ siècle, avec l'installation de la Compagnie de pulpe de Chicoutimi par Alfred Dubuc. Le site qui rassemble les plus importants vestiges de l'histoire industrielle de la région a été converti en musée. La Pulperie permet aux visiteurs de découvrir une partie de l'histoire tout en admirant l'impétuosité de la rivière Chicoutimi et les magnifiques édifices de pierre qui la bordent. En outre, les nouveaux paysages qui découlent des aménagements réalisés sur les espaces libérés par la catastrophe convient à la redécouverte du quartier du Bassin.

Au cours des dernières années, l'amélioration des paysages urbanisés s'est poursuivie. La sensibilité accrue des citadins pour leur environnement a incité les municipalités du Saguenay à se doter d'équipements de qualité. L'aménagement du Vieux Port de Chicoutimi en un vaste espace récréatif a redonné un accès direct à la rivière Saguenay et à des paysages qui annoncent déjà le fjord. La ville de Jonquière, avec le parc de la rivière aux Sables, possède une belle et longue promenade qui a rehaussé la qualité de ses paysages urbains tout en rendant accessibles ses vieux quartiers. Quant au parc Mars à La Baie, il fournit aux citoyens et aux visiteurs l'occasion de se réapproprier les paysages grandioses de la Baie des $\mathrm{Ha}$ ! $\mathrm{Ha}$ ! Il est intéressant de souligner qu'on se préoccupe de plus en plus de l'intégration des composantes du paysage dans la conception des nouveaux aménagements où se mélangent nature et culture.

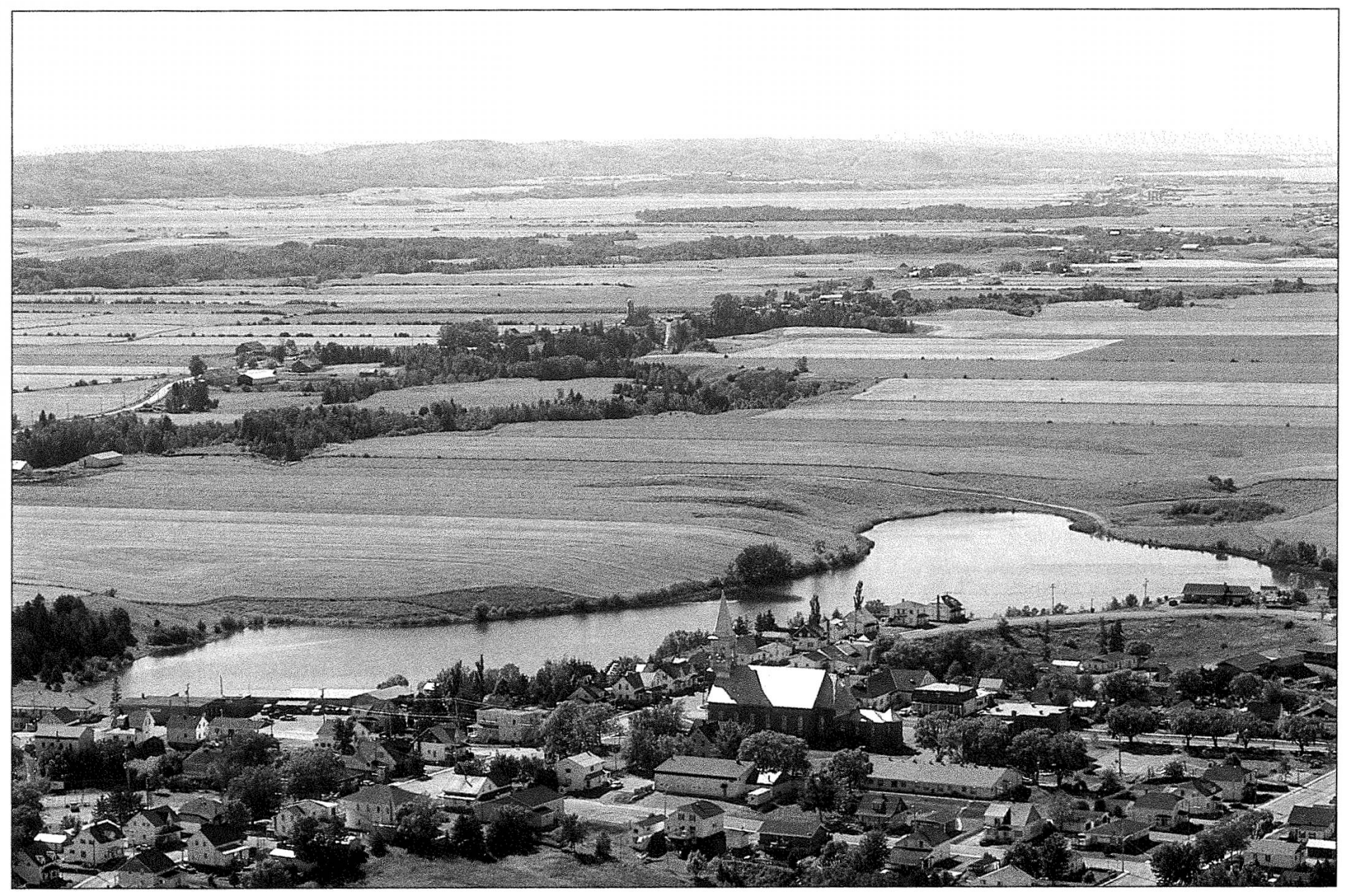

Lac-à-la-Croix, au Lac-Saint-Jean, photo Pierre Lahoud. 
Ce bref tour d'horizon serait incomplet sans une petite escapade en périphérie des zones urbaines où la nature reprend rapidement ses droits. Au nord, le massif des Monts Valin, que l'on aperçoit de presque partout au Saguenay, surplombe la région du haut de ses 1000 mètres d'altitude. C'est une destination reconnue pour les sports d'hiver et ses paysages féeriques d'arbres momifiés. Au sud, le lac Kénogami, grand lac de 27 kilomètres de long, présente des paysages plutôt contrastés. Le nouveau sentier de randonnée reliant Hébertville à Laterrière donne accès à des points de vue inédits où s'entremêlent le massif des Laurentides et le lac Kénogami.

\section{LES PAYSAGES DE GRANDE NATURE DU FJORD DU SAGUENAY}

Le fjord du Saguenay se caractérise par un ensemble unique de paysages naturels. $\mathrm{Sa}$ situation géographique en fait l'un des fjords les plus méridionaux au monde. Cette vallée glacière, creusée et rabotée par le passage successif des glaciers, est souvent très accidentée. Des parois rocheuses et des falaises abruptes de plus de 300 mètres de hauteur surplombent le Saguenay, offrant des panoramas qui portent à plusieurs kilomètres de distance. De Saint-Fulgence à Tadoussac, le spectacle est continu et toujours grandiose.

À l'extrémité du Cap-des-Roches, la flèche littorale de Saint-Fulgence s'étire sur une distance de 650 mètres. Elle marque la limite ouest du fjord du Saguenay. Il s'agit d'un des rares exemples, au Québec, d'une accumulation de matériaux dont la position est perpendiculaire au rivage. En amont de la flèche (rivière Saguenay), la profondeur de l'eau à marée basse est inférieure à 6 mètres, alors que de l'autre côté de la flèche (fjord du Saguenay), elle augmente pour atteindre 80 mètres. Une halte aux battures de Saint-Fulgence permet d'établir un premier contact avec la richesse floristique et aviaire de cet estuaire maritime. La diversité des conditions (apport d'eau douce, eau salée, marées et courants) favorise la croissance d'une flore abondante et la présence d'une grande variété d'oiseaux. On y a inventorié audelà de 175 espèces de plantes et recensé plus de 240 espèces d'oiseaux. SaintFulgence accueille même, à l'occasion, le béluga, seul cétacé qui fréquente le fjord.
On a créé deux parcs pour protéger et rendre accessible ce milieu naturel exceptionnel que constitue le fjord. Le premier, le parc du Saguenay, est reconnu pour la qualité de son circuit de randonnée pédestre. Le tracé des sentiers offre une grande intimité avec les paysages sublimes des caps, des falaises et des montagnes qui entourent le fjord. Le sentier de la Statue, qui serpente doucement les flans en granit du cap Éternité, permet d'atteindre la célèbre statue de la Vierge, érigée en 1881, qui porte son regard loin à l'horizon et veille sur les navigateurs empruntant les eaux souvent tumultueuses du Saguenay où les marées atteignent une moyenne de 4,3 mètres. La création du parc marin Saguenay-Saint-Laurent vise à assurer la diversité biologique de cette aire marine et à faire connaître les phénomènes océanographiques particuliers qui s'y produisent. L'envahissement du fjord par les eaux salées du Saint-Laurent, la présence de trois immenses bassins, les variations de température et de salinité en font un milieu presque aussi riche que l'estuaire du Saint-Laurent. En effet, on y trouve 59 espèces de poissons comparativement à 74 dans l'estuaire. La pêche blanche, qui se pratique principalement à $\mathrm{La}$ Baie et à Sainte-Rose-du-Nord, réserve souvent des surprises à ses adeptes. Il arrive qu'un requin du Groenland prenne la place des sébastes, des morues et des flétans qui constituent le lot habituel des pêcheurs.

Les traces de l'occupation humaine sont éparpillées, ici et là, le long du fjord du Saguenay. Elles se concentrent autour d'étroites vallées enserrées à l'intérieur d'immenses étendues de forêt. C'est à partir du milieu du XIX ${ }^{\mathrm{e}}$ siècle qu' a commencé le peuplement des premiers villages. À cette époque, la navigation constituait le seul moyen de transport et de communication avec l'extérieur. Les excursions, à bord des nombreux bateaux de croisière qui sillonnent le fjord, de Chicoutimi à Tadoussac, procurent des sensations qui s'apparentent à celles des premiers colons venus, en goélette, à la découverte et à la conquête du Saguenay. On pénètre dans un environnement au relief tourmenté exprimant l'impétuosité des forces de la nature. L'omniprésence de la pierre et du roc ainsi que la beauté sauvage des paysages suscitent admiration et surprise.

La route a réduit le sentiment d'isolement des petites communautés où prédominent les activités forestières. Les quelques espaces agricoles qu' on y trouve contribuent à diversifier les paysages et à mettre en valeur le patrimoine bâti. Les éléments d'intérêt fourmillent et plusieurs villages offrent de belles ouvertures sur les paysages du fjord. Sur la rive sud, les villages de L'Anse-Saint-Jean et de Petit-Saguenay proposent des sentiers et des belvédères qui offrent des scènes inoubliables. Les rivières à saumons, les escarpements rocheux, le sommet des montages composent des arrangements grandioses où viennent se joindre les composantes humaines caractéristiques du milieu (ponts couverts, quais, etc.). L'Anse-à-la-Croix où s'est implanté « Le site de la NouvelleFrance » offre également une vue magistrale sur la baie des $\mathrm{Ha}$ ! $\mathrm{Ha}$ ! Sur la rive nord, le pittoresque village de Sainte-Rosedu-Nord propose des panoramas remarquables. De là, on peut emprunter la route 172 qui longue la magnifique rivière SainteMarguerite. Le trajet consiste en un enchaînement de paysages où chaque détour dévoile les attraits de cette vallée étroite et profonde. Tout itinéraire mène invariablement à Tadoussac, l'une des plus belles baies du Québec. Ce haut lieu de l'histoire de l'Amérique constitue la porte d'entrée du fjord avec sa topographie saisissante et ses paysages glaciaires.

Jocelyn Caron est directeur-coordonnateur du Conseil du loisir scientifique du Saguenay-LacSaint-Jean, organisme de promotion du loisir et de la culture scientifiques qui s'intéresse, entre autres, au tourisme scientifique et à l'interprétation des paysages. Il a produit, au cours de la dernière année, un Inventaire préliminaire des paysages du Saguenay-Lac-Saint-Jean et procède actuellement à la conception d'une grille descriptive pour en faire la caractérisation.

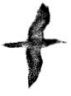

\section{BIBLIOGRAPHIE}

Bédard, Renée, Jocelyn Caron et coll. (1999), Le lac Saint-Jean, portrait d'une mer intérieure, Conseil du loisir scientifique du Saguenay-LacSaint-Jean et le Groupe MADIE, 64 p.

Conseil du loisir scientifique du Saguenay-LacSaint-Jean (1999), Inventaire préliminaire des paysages du Saguenay-Lac-Saint-Jean, Alma, $83 \mathrm{p}$.

Dumas, Alain, et Yves Ouellet (1999), Saguenay-Lac-Saint-Jean, Un royaume au Québec, Éditions du Trécarré, 159 p.

Robitaille, André, et Jean-Pierre Saucier (1998), Paysages régionaux du Québec méridional, Québec, Les Publications du Québec, 213 p. 